\title{
The Dark Halo - Spheroid Conspiracy Reloaded: Evolution with Redshift
}

\author{
Rhea-Silvia Remus ${ }^{1}$, Klaus Dolag ${ }^{1,2}$ and Andreas Burkert ${ }^{1,3}$ \\ ${ }^{1}$ Universitäts-Sternwarte München, Scheinerstr. 1, D-81679 München, Germany \\ ${ }^{2}$ MPI for Astrophysics, Karl-Schwarzschild Strasse 1, D-85748 Garching, Germany \\ ${ }^{3}$ MPI for Extraterrestrial Physics, Giessenbachstrasse 1, D-85748 Garching, Germany \\ email: rhea@usm.lmu.de
}

\begin{abstract}
The total density profiles of elliptical galaxies can be fit by a single power law, i.e., $\rho_{\text {tot }} \propto r^{\gamma}$ with $\gamma \approx-2$. While strong lensing observations show a tendency for the slopes to become flatter with increasing redshift, simulations indicate an opposite trend. To understand this discrepancy, we study a set of simulated spheroids formed within the cosmological framework. From our simulations we find that the steepness of the total density slope correlates with the compactness of the stellar component within the half-mass radius, and that spheroidal galaxies tend to be more compact at high redshifts than their present-day counterparts. While both these results are in agreement with observations, the observed trend of the total density slope with redshift remains in contradiction to the results from simulations.
\end{abstract}

Keywords. galaxies: elliptical - galaxies: evolution - galaxies: formation

\section{Introduction}

At low redshifts, elliptical galaxies are known to consist mostly of an (old) spheroidal stellar component embedded in a dark matter halo. The transition zone, where the dark matter starts to dominate over the stellar component, provides information about the galaxies' formation history; observationally, however, the kinematics in this area are difficult to trace. For spiral galaxies it is long known from observations that the rotation curves are flat out to large radii (Einasto et al. 1974, Faber \& Gallagher 1979), which is called the dark-halo-disk conspiracy, and detailed dynamical modeling (e.g., Gerhard et al. 2001) has demonstrated more recently that between the dark halo and the spheroid of massive, slow rotating elliptical galaxies a similar conspiracy might exist. This conspiracy for spheroidal galaxies has been studied from simulations in detail by Remus et al. (2013) (hereafter R13) for a sample of ellipticals from binary mergers and cosmological zoom-in simulations. They find that the total density and velocity dispersion profiles of spheroidal galaxies can be described by single power laws, with slopes of $\gamma \approx-2$ for the density and $\gamma \approx 0$ for the velocity dispersion. They also report a strong trend towards steeper slopes with redshift.

One important observational method for studying the total density profiles of elliptical galaxies is strong lensing: For low redshift ellipticals, the SLACS survey finds that the total density profiles can be described by a power law with a slope of $\gamma \approx-2$ (Auger et al. 2010, Barnabè et al. 2011), with the steepness of the slope correlating with the central density of the stellar component and the effective radius, which agrees well with the result from $\mathrm{R} 13$. However, for higher redshifts up to $z \approx 1$, strong lensing studies report a slight trend towards flatter slopes (Treu \& Koopmans 2004, Ruff et al. 2011, Bolton et al. 2012, Sonnenfeld et al. 2013) in contradiction to the trends found by R13. We present a brief preview of a more detailed study on the evolution of the dark-halo-spheroid conspiracy with redshift and its implications for the formation of elliptical galaxies (Remus et al., in 

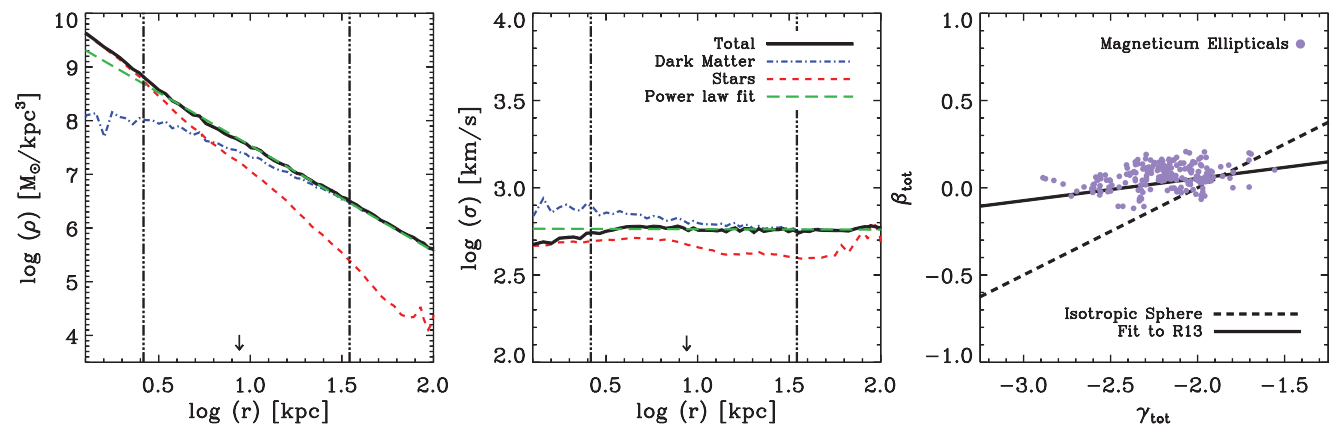

Figure 1. Left: Density profiles for a spheroidal from Magneticum: total combined stellar and dark matter profile (black solid line), stellar component (red dashed line), dark matter component (blue dash-dotted line), and a power-law fit to the total density profile (green long-dashed line). The arrow marks $R_{1 / 2}$. Center: Velocity dispersion profile for the same spheroidal. Colors as in the left panel. Right: Slopes of the total velocity dispersion profiles $\beta_{\text {tot }}$ vs. those of the total density profiles $\gamma_{\text {tot }}$ for all spheroidals selected from the simulation. Dashed black line: solution of the Jeans Equation for an isotropic sphere in hydrostatic equilibrium. Solid black line: fit to the zoom-in simulations from R13.

prep.), using spheroidals selected from a full hydrodynamic cosmological box with high resolution called Magneticum Pathfinder (Dolag et al., in prep.).

\section{The Magneticum Pathfinder Simulations}

The Magneticum Pathfinder simulations* (Dolag et al., in prep.) are a set of hydrodynamical cosmological boxes with different resolutions $\left(m_{\mathrm{Gas}}=2.6 \cdot 10^{9} M_{\odot} / \mathrm{h}\right.$ up to $\left.m_{\mathrm{Gas}}=3.9 \cdot 10^{5} \mathrm{M}_{\odot} / h\right)$ and volumes covering $(896 \mathrm{Mpc} / h)^{3}$ to $(18 \mathrm{Mpc} / h)^{3}$, performed with a modified version of GADGET (Springel 2005). They include state-ofthe-art physics, especially black hole feedback, and improvements for smooth particle hydrodynamics (for detailed descriptions see Hirschmann et al. 2014, Dolag et al. 2005, Dolag et al., in prep., and Beck et al., in prep.). We adopt a $\Lambda$ CDM cosmology according to WMAP7 (Komatsu et al. 2011) with $h=0.704, \Omega_{m}=0.272$ and $\Lambda=0.728$, and use SUBFIND (Springel et al. 2001, Dolag et al. 2009) to identify and extract structures.

We use the largest Magneticum box with enough resolution to study the dynamics of spheroidal galaxies in detail. It has a box length of $48 \mathrm{Mpc} / h$ and a resolution of $m_{\mathrm{DM}}=3.6 \cdot 10^{7} M_{\odot} / h, m_{\mathrm{Gas}} \approx 7.3 \cdot 10^{6} M_{\odot} / h$, and slightly smaller stellar mass, with $(2 \cdot 576)^{3}$ particles and a softening length of $\epsilon_{\mathrm{DM}, \mathrm{Gas}}=1.4 \mathrm{kpc} / h$ for dark matter and gas and $\epsilon_{*}=0.7 \mathrm{kpc} / h$ for stars. This simulation successfully reproduces populations of disk as well as spheroidal galaxies. To ensure sufficient resolution, we exclude galaxies with stellar masses less than $M_{*}=1 \cdot 10^{11} M_{\odot}$. We use a procedure similar to Scannapieco et al. (2008) to select spheroidals without cold gas disks from the sample of galaxies (see Remus et al. (2014) for more details). A more advanced study including spheroidals with small gas disks and S0-galaxies is in preparation (Remus et al.).

\section{The Dark Halo - Spheroid Conspiracy with Redshift}

The total density and velocity dispersion profiles of spheroidal galaxies can be fit by single power laws within a range of $0.3 R_{1 / 2}$ to $4 R_{1 / 2}$, as shown by $\mathrm{R} 13$ for a set of cosmological zoom-in simulations and isolated binary mergers. The inner parts of

*www.magneticum.org 


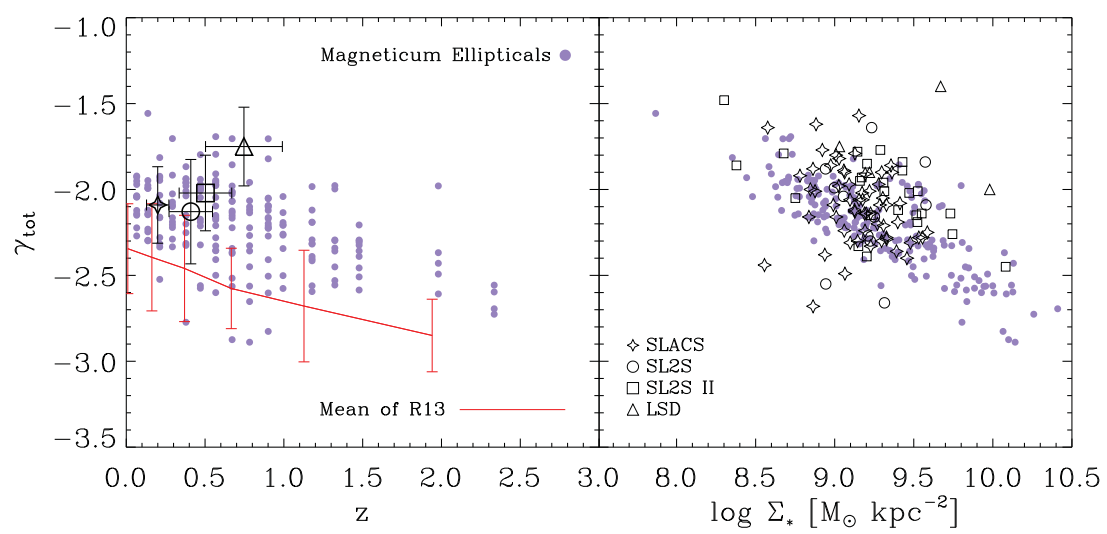

Figure 2. Left: Total density slope versus redshift. Filled lilac circles are the Magneticum spheroidals. The red solid line marks the mean of the values from R13, the open symbols the means of the observations from SLACS (diamonds, Auger et al. 2010 and Barnabè et al. 2011), SL2S (circles from Ruff et al. 2011, squares from Sonnenfeld et al. 2013) and LSD (triangles, Treu \& Koopmans 2004), with the error bars the square roots of the variance. Right: Total density slope versus stellar mass density. Symbols and colors as in the left panel, but with the open symbols representing the individual observations from the different surveys.

the total profiles are dominated by the stellar component, while in the outer parts the dark matter contributes most to the profiles. We find the same behaviour for the Magneticum spheroidals, as demonstrated in the left and central panel of Fig. 1 for an example spheroidal. The slopes of the power-law fits,

$$
\beta=\frac{\mathrm{d} \log (\sigma)}{\mathrm{d} \log (r)} \quad \text { and } \quad \gamma=\frac{\mathrm{d} \log (\rho)}{\mathrm{d} \log (r)},
$$

are shown in the right panel of Fig. 1. We find that the slopes do not follow the solution of the Jeans Equation for an isotropic sphere (or a sphere with constant anisotropy) in hydrostatic equilibrium assuming a single power-law solution for both density and velocity dispersion, but show constant values for $\beta$, while $\gamma$ covers the whole range between -3 and -1.5 . This is in agreement with the results from $\mathrm{R} 13$, who showed that $\beta$ is constant independent of the spheroidals' mass, size, in-situ or central dark matter fraction, while the steepness of the total density profiles correlates with the amount of stars formed in-situ and anticorrelates with the dark matter fraction.

However, while R13 found a steepening of $\gamma$ with redshift, observations appear to indicate a different behaviour. The left panel of Fig. 2 shows $\gamma$ for the Magneticum spheroidals for different redshifts, together with the observed trends and the results from R13. While the steepening of $\gamma$ with redshift is not as strong as for the galaxies in R13, we still find the tendency towards steeper slopes for higher redshifts, in contradiction to observations. The difference between the two simulated samples of galaxies is partly due to the improvements to SPH and the additional physics included in the Magneticum simulations, which cause the galaxies to be more realistic (see, for example, the masssize relation shown by Remus et al. 2014), but primarily an effect of the mass selection criterion applied to the Magneticum samples: while R13 followed a set of 20 galaxies back in time, the Magneticum sample includes spheroidals above $M_{*}=1 \cdot 10^{11} M_{\odot}$ independent of their progenitors and thus includes more massive systems at high redshifts than the sample studied by R13. The right panel of Fig. 2 shows the dependence of $\gamma$ on the stellar mass density $\Sigma_{*}=M_{*} / 2 \pi R_{1 / 2}^{2}$, following Sonnenfeld et al. (2013). Our Magneticum spheroidals are in very good agreement with the observations, with a clear 
trend towards steeper slopes for more compact spheroids. Since spheroidals at higher redshifts are generally more compact than their present day counterparts in mass (e.g., van de Sande et al. 2013), this supports the idea that the total density slopes $\gamma$ should be generally steeper with redshift. Therefore, we conclude that it is crucial to understand the origin of the differences between the observed and simulated trends of the total density slope with redshift. A detailed study on this subject will be presented in a forthcoming paper by Remus et al. (in prep.).

\section{Conclusions}

The total combined density profiles of Magneticum spheroidal galaxies can be fit by a single power law with a slope of on average $\gamma \approx-2$, with a tendency towards steeper slopes for more compact ellipticals. At higher redshifts, the density slopes of our spheroidals are generally steeper, in agreement with results presented in R13. While observations agree with the simulations, that spheroidal galaxies tend to be more compact at high redshifts than their present-day counterparts, the observed flattening of the total density slope with redshift contradicts the results from both the simulations presented in R13 and the Magneticum spheroidals presented in this work. The origin of this different behaviour will be discussed in a forthcoming paper by Remus et al. (in prep.). In addition, we showed that the slope of the total velocity dispersion is constant independent of the stellar mass density and the total density slopes, in agreement with simulations by R13. This behaviour is still an unsolved problem, and will be subject to further investigations.

\section{Acknowledgements}

The authors acknowledge support from the DFG Excellence Cluster Universe, the Computational Center C2PAP, and the Leibniz-Rechenzentrum via projects pr86re and pr83li.

\section{References}

Auger, M. W., et al. 2010, ApJ, 724, 511

Barnabè, M., et al. 2011, MNRAS, 415, 2215

Bolton, A. S., et al. 2012, ApJ, 757, 82

Dolag, K., Vazza, F., Brunetti, G., \& Tormen, G. 2005, MNRAS, 364, 753

Dolag, K., Borgani, S., Murante, G., \& Springel, V. 2009, MNRAS, 399, 497

Einasto, J., Kaasik, A., \& Saar, E. 1974, Nature, 250, 309

Faber, S. M. \& Gallagher, J. S. 1979, ARA\& A, 17, 135

Gerhard, O., Kronawitter, A., Saglia, R. P., \& Bender, R. 2001, AJ, 121, 1936

Hirschmann, M., et al. 2014, MNRAS, 442, 2304

Komatsu, E., et al. 2011, ApJS, 192, 18

Remus, R.-S., Burkert, A., \& Dolag, K., et al. 2013, ApJ, 766, 71

Remus, R.-S., et al. 2014, in IAU Symp. 309, Galaxies in 3D across the Universe, eds. B. L. Ziegler, F. Combes H. Dannerbauer \& M. Verdugo, in press

Ruff, A. J., et al. 2011, ApJ, 727, 96

Scannapieco, C., et al. 2008, MNRAS, 389, 1137

Sonnenfeld, A., Treu, T., Gavazzi, R., et al. 2013, ApJ, 777, 98

Springel, V. 2005, MNRAS, 364, 1105

Springel, V., White, S. D. M., Tormen, G., \& Kauffmann, G. 2001, MNRAS, 328, 726

Treu, T. \& Koopmans, L. V. E. 2004, ApJ, 611, 739

van de Sande, J., Kriek, M., Franx, M., et al. 2013, ApJ, 771, 85 